\title{
Analytic inversion formula for confocal scanning microscopy
}

\author{
B. Bertero \\ Dipartimento di Fisica dell' Università and Istituto Nazionale di Fisica Nucleare, I-16146 Genova, Italy \\ C. De Mol \\ Département de Mathématique, Université Libre de Bruxelles, B-1050 Bruxelles, Belgium \\ E. R. Pike \\ Department of Physics, King's College London (KQG), Strand, London, England \\ Received January 22, 1987; accepted May 4, 1987
}

\begin{abstract}
We find a simple analytic expression for the inverse of an operator related to the problem of data reduction in confocal scanning microscopy. Potential applications of this result to the practical scanning-microscope problem are outlined.
\end{abstract}

In two recent papers ${ }^{1,2}$ we proposed a method for enhancing resolution in confocal scanning microscopy. ${ }^{3}$ First we summarize the main points of the procedure in the case of a onedimensional optical system with ideal lenses. Let $f(y)$ be the complex effective transparency (or reflectivity for a reflection microscope) in the object plane; by this we mean that, for a given scanning position, the image $g(x)$ formed by an ideal microscope with a uniformly filled illumination lens is

$$
g(x)=\int_{-\infty}^{+\infty} \operatorname{sinc}(x-y) \operatorname{sinc}(y) f(y) \mathrm{d} y,
$$

where $\operatorname{sinc}(x)=\sin (\pi x) /(\pi x)$. The basic idea, then, is to record the whole image $g(x)$ at each scanning position and to solve the integral equation (1) for $f(y)$. In Ref. 2 it was shown that if $g(x)$ is detected at only a small number of points sampled at the Nyquist (Rayleigh) distance, and if the method of truncated singular function expansions is used for recovering $f(y)$, then a considerable improvement is obtained with respect to the image formed by the usual type II scanning microscope in which only the central point of the image is recorded. ${ }^{3}$

The integral operator involved in Eq. (1) has convenient mathematical properties. First, the orthogonal complement of its null space is the set of all the functions $f$ with bandwidth $2 \pi$ that have zeros at the integer sampling points. 4 The projection of an arbitrary $f$ on this subspace we call the transmitted part of $f$. Since the component of $f$ on the null space, i.e., the invisible component of $f$, is completely lost, we can assume, without loss of generality, that $f$ coincides with its transmitted part so that it can be represented by the following form:

$$
f(y)=\sum_{m \square-\infty}^{+\infty} f\left(y_{m}\right) \operatorname{sinc}\left[2\left(y-y_{m}\right)\right],
$$

where

$$
y_{0}=0, \quad y_{m}=\operatorname{sgn}(\mathrm{m})(|m|-1 / 2), \quad m= \pm 1, \pm 2, \ldots
$$

A second important result, obtained recently by Gori and Guattari, ${ }^{4}$ is that one can find simple analytic expressions for the singular values $\alpha_{k}$ and singular functions $u_{k}$ and $v_{k}$ of the integral operator [Eq. (1)]. This result, of course, permits a series representation of the generalized inverse of the operator [Eq. (1)], since the solution of minimal norm of Eq. (1) is given by

$$
f(y)=\sum_{k=0}^{+\infty} \frac{1}{\alpha_{k}}\left(g, v_{k}\right) u_{k}(y) .
$$

Here, $\left(g, v_{k}\right)$ denotes the usual scalar product in $L^{2}(-\infty,+\infty)$.

As shown in Ref. 2, if we use the sampling expansion for the (noise-free) image $g$,

$$
g(x)=\sum_{n=-\infty}^{+\infty} g(n) \operatorname{sinc}(x-n),
$$

and the expansion [Eq. (2)] for the transmitted part of $f$, then Eq. (1) can be transformed into an infinite-dimensional linear system given by

$$
g(n)=\sum_{m=-\infty}^{+\infty} A_{n m} f\left(y_{m}\right)
$$

where

$$
A_{n o}=1 / 2 \delta_{n o}, \quad n=0, \pm 1, \ldots
$$

and

$$
\begin{aligned}
A_{n m}=\frac{1}{2 \pi^{2}} \frac{(-1)^{n+1}}{y_{m}\left(n-y_{m}\right)}, \quad m & = \pm 1, \pm 2, \ldots, \\
n & =0, \pm 1, \ldots .
\end{aligned}
$$

Notice that, although the integral operator [Eq. (1)] does not have an inverse but only a generalized inverse, the infinite- 
dimensional matrix $A$ given by Eqs. (7) is invertible, since it is equivalent to the restriction of the integral operator [Eq. (1)] to the orthogonal complement of its null space.

We will prove in this paper that the inverse matrix $A^{-1}$ has the following simple expressions:

$$
\begin{aligned}
\left(A^{-1}\right)_{o n}=2(-1)^{n}, & n=0, \pm 1, \ldots, \\
\left(A^{-1}\right)_{m n}=(-1)^{n+1} \frac{2 n}{n-y_{m}}, & m= \pm 1, \pm 2, \ldots, \\
n & =0, \pm 1, \ldots .
\end{aligned}
$$

Once these results are found, the fact that they satisfy $A^{-1} A$ $=A A^{-1}=I$ can easily be checked by direct computations, using values of standard numerical series.

The starting point of the derivation is the fact that the matrix elements of the inverse operator $A^{-1}$ can be expressed in terms of the singular system of the integral operator [Eq. (1)]. The result is

$$
\left(A^{-1}\right)_{m n}=\sum_{k-0}^{+\infty} \frac{1}{\alpha_{k}} u_{k}\left(y_{m}\right) v_{k}(n),
$$

which follows easily from Eq. (4) and the remark that $\left(g, v_{k}\right)$ $=\sum_{n} g(n) v_{k}(n)$.

Now we use the results of Gori and Guattari in order to transform the series in Eq. (9). This can be split into two parts, the first containing the odd values of $k$ and the second containing the even values of $k$ (corresponding to odd and even singular functions, respectively). In the case $m=0$, all the odd terms are zero, while in the case $m \neq 0$ only one odd term is nonzero, namely, that corresponding to $k=2|m|-1$, as can be derived from the expression of $u_{k}$ given in Ref. 4 .

The contribution of the even terms may be expressed as a series whose coefficients are a simple rational function of the zeros, $\beta_{2 j}$, of the equation $\tan (z / 2)=2 / z$. We need to introduce here the function

$$
\phi(z)=\left[\tan \left(\frac{z}{2}\right)-\frac{2}{z}\right]^{-1},
$$

which has first-order poles at the points $z= \pm \beta_{2 j}(j=0,1$, $2, \ldots$ ) with residues

$$
R_{2 j}=2 \beta_{2 j}{ }^{2}\left(8+\beta_{2 j}{ }^{2}\right)^{-1} .
$$

After some lengthy but elementary computations, using the explicit expressions of $\alpha_{\mathrm{k}}, u_{k}$, and $v_{\mathrm{k}}$ given in Ref. 4, one finds finally the following series expressions for the inverse matrix elements:

$$
\begin{aligned}
&\left(A^{-1}\right)_{o n}= 8(-1)^{n} \sum_{j=0}^{+\infty} \frac{R_{2 j}}{\beta_{2 j}{ }^{2}-4 \pi^{2} n^{2}}, \\
&\left(A^{-1}\right)_{m n}= 2(-1)^{n+1} \frac{n y_{m}}{n^{2}-y_{m}{ }^{2}}+8(-1)^{n+1} \\
& \times \sum_{j=0}^{+\infty} \frac{\beta_{2}{ }^{2} R_{2 j}}{\left(\beta_{2 j}{ }^{2}-4 \pi^{2} n^{2}\right)\left(\beta_{2 j}{ }^{2}-4 \pi^{2} y_{m}{ }^{2}\right)}, \\
& m \neq 0 .
\end{aligned}
$$

In Eq. (12b) the first term is the contribution of the odd term.
These series can now be computed by contour integration. If $\Gamma$ is a circle of large radius that crosses the real axis between two poles of the function $\phi(z)$, and if one integrates the function

$$
\phi(z)\left(z^{2}-4 \pi^{2} n^{2}\right)^{-1}
$$

in the case (12a) and the function

$$
z^{2} \phi(z)\left(z^{2}-4 \pi^{2} n^{2}\right)^{-1}\left(z^{2}-4 \pi^{2} y_{m}{ }^{2}\right)^{-1}
$$

in the case (12b) over this circle, then it is easy to see that the value of these integrals tends to zero when the radius of the circle tends to infinity. These integrals can, alternatively, be evaluated by means of the residue theorem, by evaluating the sum of the contributions of the poles at $z= \pm \beta_{2 j}$ and $z=$ $\pm 2 \pi n$, and this sum must therefore also vanish. Notice that the function (13b) does not have poles at $z= \pm 2 \pi y_{m}$. In this way one obtains the following results:

$$
\begin{gathered}
\sum_{j=0}^{+\infty} \frac{R_{2 j}}{\beta_{2 j}{ }^{2}-4 \pi^{2} n^{2}}=1 / 4, \\
\sum_{j=0}^{+\infty} \frac{\beta_{2 j}{ }^{2} R_{2 j}}{\left(\beta_{2 j}{ }^{2}-4 \pi^{2} n^{2}\right)\left({\beta_{2 j}}^{2}-4 \pi^{2} y_{m}{ }^{2}\right)}=\frac{n^{2}}{4\left(n^{2}-y_{m}{ }^{2}\right)},
\end{gathered}
$$

which, combined with Eqs. (12a) and (12b), give Eqs. (8a) and $(8 \mathrm{~b})$ for the inverse matrix.

We comment now on the special case $m=0$, which is relevant when one wishes to recover the function $f(y)$ only on the optical axis, $y=0 .^{2}$ From Eqs. (4) and (8a) we find that

$$
f(0)=2 \sum_{n=-\infty}^{+\infty}(-1)^{n} g(n)
$$

A simple derivation of this special case can be obtained from the sampling expansion of the cosine,

$$
\cos (\pi x)=\sum_{n=-\infty}^{+\infty}(-1)^{n} \operatorname{sinc}(x-n) .
$$

We have from Eq. (1)

$$
\begin{aligned}
2 \sum_{n=-\infty}^{+\infty}(-1)^{n} g(n)= & 2 \sum_{n=-\infty}^{+\infty}(-1)^{n} \\
& \times \int_{-\infty}^{+\infty} \operatorname{sinc}(n-y) \operatorname{sinc}(y) f(y) \mathrm{d} y \\
= & 2 \int_{-\infty}^{+\infty} \operatorname{sinc}(y) \cos (\pi y) f(y) \mathrm{d} y \\
& =2 \int_{-\infty}^{+\infty} \operatorname{sinc}(2 y) f(y) \mathrm{d} y=f(0)
\end{aligned}
$$

where, in the last step, the assumption that $f(y)$ is band limited with bandwidth $2 \pi$ and the projection property of the sinc kernel have been used.

It is interesting to notice that by numerical observation, the simple exact inversion formula [Eq. (15)] is well approximated even when one uses the singular system corresponding to a small number of data points. In fact, if we use $2 N+$ 1 data points, then the corresponding recovered value of $f(0)$, given by the method developed in Ref. 2 , is 


$$
f(0)=\sum_{n=-N}^{N} t_{n} g(n),
$$

where

$$
t_{n}=\sum_{k=0}^{2 M} \frac{1}{\alpha_{N, k}} v_{N, k}(n) u_{N, k}(0),
$$

$2 M+1$ is the number of singular values used $(M<N)$, and $\alpha_{N, k}, u_{N, k}, v_{N, k}$ is the singular system of the problem with $2 N$ +1 data (see Ref. 2). Using only five data points (as in Ref. 2 ) and five singular values, we have found $t_{n} \simeq 1.85(-1)^{n}$ with variations of the fourth digit only. This numerical observation was, in fact, the starting point of our search for an analytic expression of the inverse of the infinite-dimensional matrix $A$.

The infinite-dimensional matrix $A^{-1}$, defined by Eqs. (8), is not continuous in $l^{2}$, the Hilbert space of the squaresummable sequences of complex numbers. This is an obvious consequence of the fact that the problem of solving Eq. (1) is ill posed. In particular, the evaluation of $f(0)$ by means of Eq. (15) is not stable. The series must be truncated. If we keep $2 N+1$ terms and the errors on the data values $g(n)$ are uncorrelated random variables with zero mean and variance $\sigma^{2}$, then the variance of the error on the sum is $(2 N+$ 1) $\sigma^{2}$ and therefore grows with the number of terms. We also have some numerical evidence that the truncation of the series in Eq. (15) is approximately equivalent to the truncation of the singular function expansion. If we use Eq. (19) for evaluating the transfer vector $t_{n}$ with $M<N$, then the value of $\left|t_{n}\right|$ with $n=0, \pm 1, \ldots, \pm M$ is approximately 2 , whereas the other components are much smaller (by a factor of 10 or more).

We conclude this paper with a few remarks about potential applications of Eq. (15). Its extension to the two-dimensional case with square pupils is obvious:

$$
f(0,0)=4 \sum_{p, q=-\infty}^{+\infty}(-1)^{p+q} g(p, q) .
$$

Thus, in this case, the inversion algorithm is extremely sim- ple, and one can contemplate an implementation to produce superresolved images in real time.

Unfortunately, Eq. (20) is true only in the case of the ideal square pupil. In the case of circular lenses with aberrations, one must replace this equation by

$$
f(0,0)=\sum_{p, q} t_{p, q} g(p, q),
$$

where the transfer matrix $t_{p, q}$ must be computed by using the singular system of the measured integral operator corresponding to the real optical system. In the case of small aberrations and small deviations from the square-pupil result, one might perhaps be able to justify a formula of the type

$$
t_{p, q}=4(-1)^{p+q}+\eta_{p, q}
$$

and compute the correction terms $\eta_{p, q}$ by means of linear perturbation theory. Such a possibility remains to be investigated.

\section{ACKNOWLEDGMENTS}

This research has been partly supported by the North Atlantic Treaty Organization under grant no. 463/84 and the European Economic Community under contract no. ST290089-3. C. De Mol is Chercheur qualifie of the Belgian National Fund for Scientific Research.

E. R. Pike is also affiliated with the Royal Signals and Radar Establishment, Saint Andrews Road, Great Malvern, Worcester WR14 3PS, United Kingdom.

\section{REFERENCES}

1. M. Bertero, C. De Mol, E. R. Pike, and J. G. Walker, "Resolution in diffraction-limited imaging. IV. The case of uncertain localization or non-uniform illumination of the object," Opt. Acta 31 , 923-946 (1984).

2. M. Bertero, P. Brianzi, and E. R. Pike, "Superresolution in confocal scanning microscopy," Inverse Probl. 3, 195-212 (1987).

3. T. Wilson and C. Sheppard, Theory and Practice of Scanning Microscopy (Academic, London, 1984).

4. F. Gori and G. Guattari, "Signal restoration for linear systems with weighted inputs," Inverse Probl. 1, 67-85 (1985). 